\title{
Reduction of Electromagnetic Force in AC Distributed Winding of Fault Current Limiter under Short-Circuit Condition
}

\author{
Asef Ghabeli $^{1 *}$, Mohammad Yazdani-Asrami ${ }^{1}$, Aref Doroudi², and S. Asghar Gholamian ${ }^{3}$ \\ ${ }^{I}$ Young Researchers and Elite Club, Sari Branch, Islamic Azad University, Sari, Iran \\ ${ }^{2}$ Faculty of Electrical Engineering, Shahed University, Iran \\ ${ }^{3}$ Electrical Machinery Laboratory, Faculty of Electrical and Computer Engineering, Babol University of Technology, Iran
}

(Received 10 June 2015, Received in final form 13 July 2015, Accepted 22 July 2015)

\begin{abstract}
Various kinds of winding arrangements can be used to enable fault current limiters (FCL) to tolerate higher forces without resulting in a substantial increase in construction and fabrication costs. In this paper, a distributed winding arrangement is investigated in terms of its effects on the short-circuit forces in a threephase FCL. The force magnitudes of the AC supplied windings are calculated by employing a finite elementbased model in the time stepping procedure. The leakage flux and radial and axial force magnitudes obtained from the simulation are compared to those obtained from a conventional winding arrangement. The comparison shows that the distributed winding arrangement significantly reduces the radial and, especially, the axial force magnitudes.
\end{abstract}

Keywords : fault current limiter, short-circuit current, finite element method, electromagnetic force

\section{Introduction}

Fault current limiters (FCL) limit fault currents to an acceptable level, so the expensive equipments and upgrades for limiting the fault can be eliminated from the network [1-4]. However, short circuit currents induce excessive forces in the FCL windings, which may cause axial or radial bending, buckling and hooping stresses and etc. Consequently, the electromagnetic forces in the FCL windings are important considerations during design, manufacturing and operation of FCLs [5, 6].

Any kind of deficiency in the strength of the winding structure due to a miscalculation in the maximum electromagnetic force may result in mechanical collapse and permanent deformation of the windings. However, it is not easy to accurately predict the transient electromagnetic force because the FCL windings have a complex structure $[3,4,7]$.

A three-phase three-legged core-type power transformer was modeled in 2-D and 3-D. The finite element method was used to calculate and compare the electromagnetic forces resulting from short-circuit and inrush current by

CThe Korean Magnetics Society. All rights reserved.

*Corresponding author: Tel: +989113510308

Fax: +989113510308, e-mail: asefghabeli@gmail.com taking into account the asymmetry of the phase current in the inrush current condition [8]. An experimental verification and finite element analysis were conducted for the electromagnetic force caused by a short circuit in a dry-type transformer. The simulation data were used to predict the stress distributions or deformations in the windings [9]. A study was also presented to calculate the mechanical force in the windings of a high-current HTS transformer with two auxiliary windings [10]. The calculations were carried out using the finite-element method by considering the non-uniformity of both the field and the current distributions. The study showed that an unbalanced current distribution may increase the mechanical forces in the windings, and an exact model to calculate the mechanical force in the windings under the aforementioned conditions was also developed.

In this paper, finite element analysis is used to calculate the electromagnetic forces on the windings of a predesigned scaled-down lab inductive FCL. First, the magnetic flux density was calculated in the FCL under normal and short-circuit conditions. Next, the axial and radial electromagnetic forces were calculated for each section of the AC supplied winding by considering two different types of winding arrangements in transient conditions. For the first winding arrangement, phase windings are placed next to each other, and in the second one, the winding 
turn of a phase is placed between the winding turns of two other phases. Finally, the radial and axial forces in these two types of winding arrangements were compared.

\section{Basic Equations}

\subsection{Transient currents}

The magnitude of the transient current could be an important factor to calculate the electromagnetic force in the FCL windings. The inductive FCL that is investigated in this paper is based on the core saturation effect. The FCL only has the capability of limiting single-phase-toground faults, and this type of fault occurs when one phase has become short-circuited to the ground, which might happen as a result of lightning strikes, debris, pollution, and animals $[5,7,11,12]$.

The electrical circuit that has been used in simulations to initiate the fault consists of a three-phase transformer that supplies a line-to-line voltage of $65 V_{r m s}$ connected to a three-phase resistive load with a resistance of $11 \Omega$ via the FCL. To initiate the fault, the load resistor in phase "c" is short-circuited at the $4^{\text {th }}$ second. This moment has been chosen to ensure that the FCL is in a steady-state condition and that the DC-supplied windings have completely driven the core legs of the FCL into saturation. The fault caused a maximum current of $395 \mathrm{~A}$ that passed through the AC-supplied windings of the FCL at the first cycle.

The parameters of the fault inception circuit are summarized in Table 1.

\subsection{Electromagnetic forces}

The current that is carried by the conductors of the FCL windings is situated in the magnetic leakage field. The fundamental principle of electromagnetic indicates that these conductors will be exposed to a force as a result of the interaction between the electric and the magnetic fields. This electromagnetic force is calculated as the vector product of the current density and the magnetic field density. A short-circuit current will have an effect on both the flux and the current densities, which means that force is proportional to the square of the current. The direction of the forces is determined by the vector product,

Table 1. Fault circuit parameters.

\begin{tabular}{cc}
\hline \hline Parameters & Value \\
\hline Line-to-line voltage $\left[V_{r m s}\right]$ & 65 \\
Fault current amplitude $\left[A_{r m s}\right]$ & 395 \\
Load resistance $[\Omega]$ & 11 \\
DC current $[\mathrm{A}]$ & 10 \\
\hline
\end{tabular}

which indicates that the force will act perpendicularly to the plane formed by the magnetic field density and the direction of the current $[5,13]$.

The electromagnetic forces in the FCL windings can be subdivided into axial and radial forces. Axial forces occur in a direction parallel to the winding height. The pattern of the leakage in the magnetic field indicates that the windings experience opposing forces at the winding ends, leading to compressive forces. The highest bending of the magnetic field occurs at the winding ends, and the maximum axial force is generated there as a consequence $[5,12]$. Radial forces occur perpendicularly to the winding height, and this kind of force occurs in almost all parts of the winding, with flux lines parallel to the winding. The direction depends on the direction in which current flows, and therefore, the force can result in a compressive or tensile stress.

\section{Distributed Winding Arrangement}

For this type of winding arrangement, the phase windings are simultaneously wound, so the winding turn of a phase is placed between the winding turns of the two other phases. This arrangement results in better magnetic coupling than when using a conventional winding arrangement, where each phase winding is wound separately. The improved magnetic coupling in the distributed winding arrangement leads to less leakage magnetic flux, and this feature results in a much lower impedance in the FCL under normal condition in the network, and consequently, much less energy loss. The other advantage of using a distributed winding arrangement over a conventional one is that a smaller electromagnetic force is applied to the AC-supplied winding during short circuits, as discussed in detail in the following sections.

\section{Modeling of the Simulated FCL}

In this paper, a pre-designed scaled-down lab FCL was modeled in 2-D with two different winding arrangements. This FCL is a three-phase inductive FCL with a common core that operates based on the core saturation effect. Fig. 1 shows the FCL modeled with two different winding arrangements.

Figure 1 shows that each of the two outer legs contains one winding for each phase. The blue, red, and green colors indicate the windings for phases $\mathrm{a}, \mathrm{b}$, and $\mathrm{c}$, respectively. Each phase winding has 9 turns and the ACsupplied windings from the same phase are connected in series. One DC-supplied winding with 40 turns, marked in black, is placed on each of the outer legs, which are 


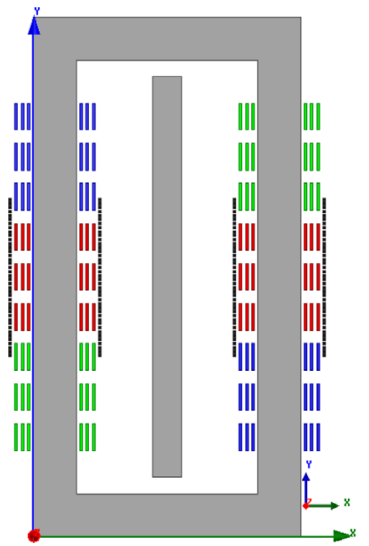

(a)

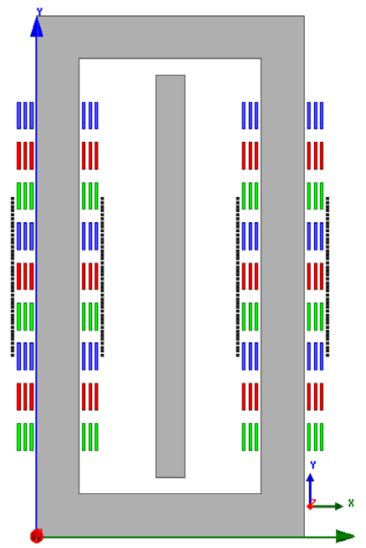

(b)
Fig. 1. (Color online) The three-phase single-core FCL modeled with the (a) phase windings placed one next to each other (Conventional) (b) Distributed phase windings.

also connected in series. Fig. 1(a) shows the conventional winding arrangement for the FCL with phase windings next to each, and Fig. 1(b) shows the distributed winding arrangement.

The specifications and dimensions of the modeled FCL are shown in Tables 2 and 3, respectively.

The AC-supplied windings were divided into 9 sections to compute the electromagnetic force. With this modeling technique, it is possible to calculate the electromagnetic forces on any particular position (or part) of the windings and to anticipate the mechanical stress, probable deformations,

Table 2. Modeled FCL Specifications.

\begin{tabular}{lc}
\hline \hline Classification & Value \\
\hline Core material & Grain-oriented \\
& silicon-steel sheets \\
Typical magnetic field intensity saturation level $[\mathrm{A} / \mathrm{m}]$ & 800 \\
Typical magnetic flux density saturation level $[\mathrm{T}]$ & 1.84 \\
Number of DC turns per leg & 40 \\
Number of AC turns per phase and per leg & 125 \\
\hline
\end{tabular}

Table 3. Modeled FCL Dimensions.

\begin{tabular}{cc}
\hline \hline Parameter & Value \\
\hline Cross-section of the outer leg $\left[\mathrm{m}^{2}\right]$ & $13.5 \times 10^{-4}$ \\
Cross-section of the middle leg $\left[\mathrm{m}^{2}\right]$ & $9 \times 10^{-4}$ \\
Core width [m] & 0.2 \\
Core height [m] & 0.41 \\
Core depth [m] & 0.042 \\
Window width [m] & 0.155 \\
Window height [m] & 0.325 \\
Width of middle leg [m] & 0.03 \\
Gap Length [m] & $2 \times 0.0125$ \\
\hline
\end{tabular}

and hazards because excessive forces are exerted on the windings. The deformations and damage mentioned above can be avoided by taking the corresponding considerations during design and manufacturing $[9,13]$.

\section{Results and Discussions}

\subsection{Simulation results}

The magnetic vector potential is computed using a magnetic field analysis, and it is used to calculate the magnetic flux density and electromagnetic forces in the FCL windings. In this paper, the parameters are processed using Maxwell v.14 which is based on the finite element method. The simulations are processed and solved using the transient solution type, the total number of mesh elements is set to 6340 , and the calculation time for each simulation is of about 30 minutes.

Figure 2 shows the distribution of the magnetic flux density for the FCL with a conventional winding arrangement [as depicted in Fig. 1(a)]. Figures 2(a) and 2(b) respectively show the magnetic flux distribution in the normal and short-circuit conditions. Figure 3 shows the distribution of the magnetic flux density for the FCL with

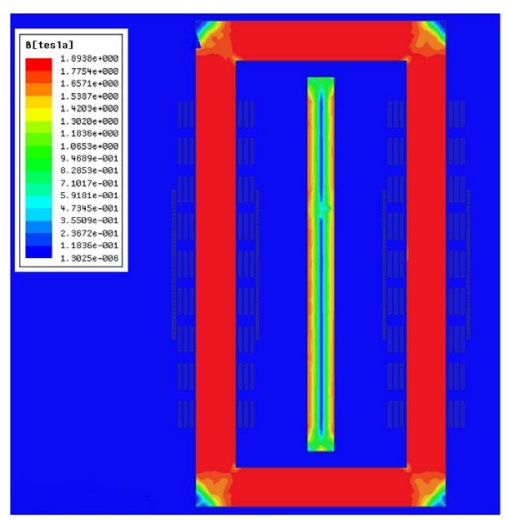

(a)

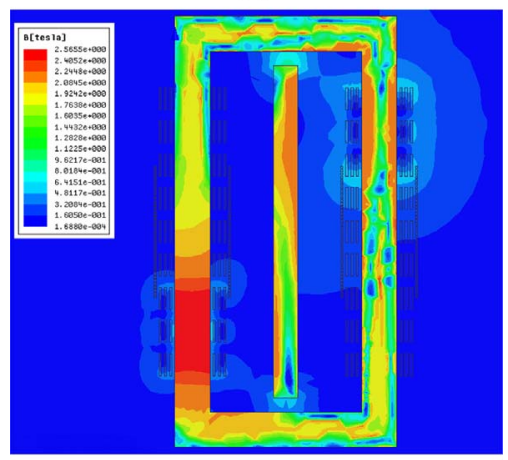

(b)

Fig. 2. (Color online) Distribution of the magnetic flux density for FCL with conventional windings (a) Saturation mode before fault (b) $20 \mathrm{~ms}$ after fault. 


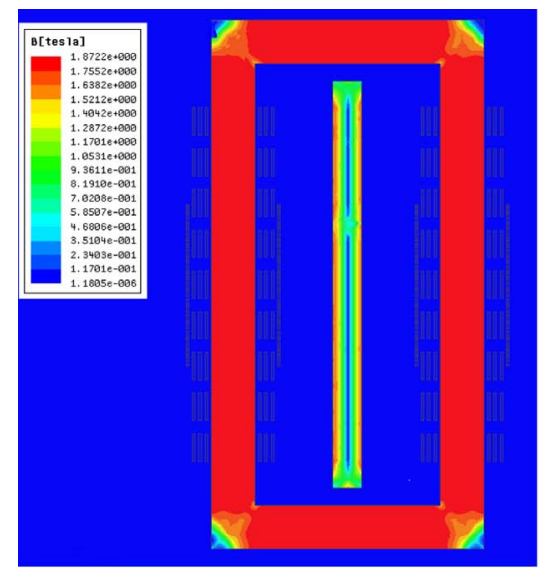

(a)

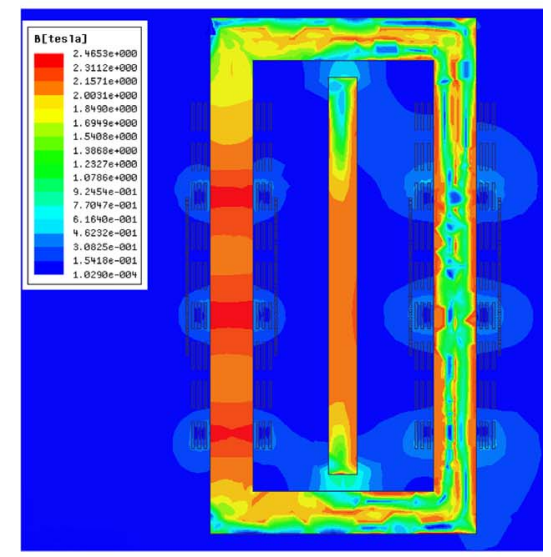

(b)

Fig. 3. (Color online) Distribution of magnetic flux density for FCL with distributed windings (a) Saturation mode before fault (b) $20 \mathrm{~ms}$ after fault.

the distributed winding arrangement [depicted in Fig. 1(b)]. Figures 3(a) and 3(b) show the magnetic flux distribution in normal and short-circuit condition, respectively. As can be seen in these figures, the short-circuit current drives one of the legs deeper into saturation in one half cycle while driving the other leg out of saturation, and this process is alternatively repeated in each half cycle. In Figures 2(b) and 3(b) the leakage flux densities around the short-circuited windings resulting from the high currents caused by the short circuit can be seen. The interactions between these leakage fluxes and high currents results in high-magnitude electromagnetic forces.

Figures 2(a) and 3(a) shows that the core saturates in steady state and that the magnetic flux density is uniformly distributed over the core in a normal condition. In Fig. 2(b), the flux lines accumulate in a region and fully drive that region into saturation when a short circuit occurs, which in turn leads to a maximum flux density of $2.56 \mathrm{~T}$. However, as seen in Fig. 3(b), the flux lines scatter all over the core for the distributed winding arrangement under short-circuit conditions, which leads to a lower maximum flux density of about $2.46 \mathrm{~T}$. This happens as a result of the particular arrangement of winding where each turn of a phase is placed between two turns of the other phases. The lower maximum flux density leads to a lower saturation, and finally to a reduction in the leakage flux and maximum electromagnetic force when compared to those of a conventional winding arrangement.

\subsection{Electromagnetic force calculations}

The electromagnetic forces, including the radial and axial forces, were accurately calculated for AC-supplied windings of FCL by using a transient solution type. The exact values of the maximum electromagnetic forces for each of the 9 sections of the AC supplied windings were obtained for a cycle after the fault inception on phase winding "c".

\subsubsection{Conventional winding arrangement}

Table 4 shows the radial and axial force magnitudes and the force magnitude for each section of conventional ACsupplied windings.

As can be seen in Table 4, the radial and axial forces in the short-circuit mode in phase winding "c" have a considerable magnitude and increase by a hundred times compared to those under normal conditions. These forces in the other two phases did not change significantly compared to those of the phase "c".

\subsubsection{Distributed winding arrangement}

After the short circuit, the electromagnetic forces, including the radial and axial forces, were also calculated for each section of the distributed AC-supplied windings, and the results were shown in Table 5.

As with the previous winding arrangement, the radial

Table 4. Radial and axial forces and force magnitude in each section of the conventional AC-supplied windings.

\begin{tabular}{ccccc}
\hline \hline Phase & Section & $\begin{array}{c}\text { Radial Force } \\
\text { Magnitude }\end{array}$ & $\begin{array}{c}\text { Axial Force } \\
\text { Magnitude }\end{array}$ & $\begin{array}{c}\text { Force } \\
\text { Magnitude }\end{array}$ \\
\hline \multirow{3}{*}{ a } & 1 & 0.24 & 1.54 & 1.56 \\
& 2 & 0.26 & 1.7 & 1.72 \\
& 3 & 0.29 & 1.95 & 1.97 \\
\hline \multirow{2}{*}{$\mathrm{b}$} & 4 & 2.7 & 6.63 & 7.16 \\
& 5 & 2.86 & 12.523 & 12.84 \\
& 6 & 1.03 & 23.53 & 23.55 \\
\hline & 7 & 94.947 & 174.5 & 198.71 \\
$\mathrm{c}$ & 8 & 109.48 & 3.145 & 109.52 \\
& 9 & 95.65 & 181.26 & 204.95 \\
\hline
\end{tabular}


Table 5. The magnitude of the radial and axial forces and force magnitude in each section of the AC-supplied windings for the distributed winding arrangement.

\begin{tabular}{ccccc}
\hline \hline Phase & Section & $\begin{array}{c}\text { Radial Force } \\
\text { Magnitude }\end{array}$ & $\begin{array}{c}\text { Axial Force } \\
\text { Magnitude }\end{array}$ & $\begin{array}{c}\text { Force } \\
\text { Magnitude }\end{array}$ \\
\hline \multirow{2}{*}{$\mathrm{a}$} & 1 & 0.16 & 2.42 & 2.43 \\
& 4 & 0.19 & 0.72 & 0.75 \\
& 7 & 0.22 & 1.81 & 1.82 \\
\hline \multirow{2}{*}{$\mathrm{b}$} & 2 & 1.46 & 23.96 & 24 \\
& 5 & 1.02 & 12.52 & 12.56 \\
\hline & 8 & 1.52 & 7.62 & 7.77 \\
\hline $\mathrm{c}$ & 3 & 75.843 & 49.28 & 90.449 \\
& 6 & 75.265 & 0.525 & 75.267 \\
\hline
\end{tabular}

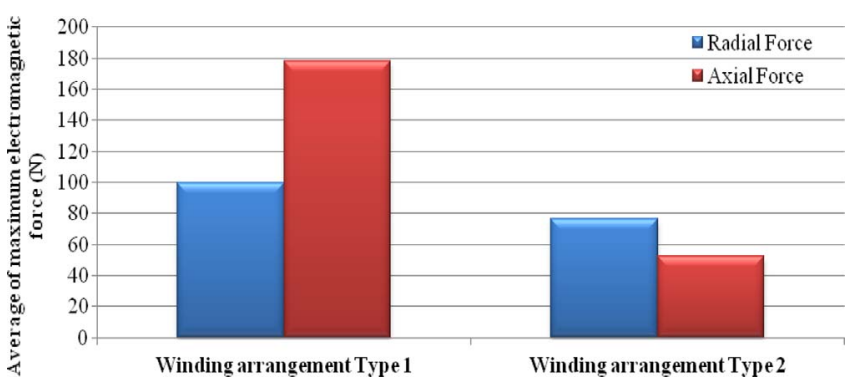

Fig. 4. (Color online) Average of the maximum electromagnetic forces of the AC-supplied windings for the simulated FCL.

and axial forces in the short-circuit mode in phase winding "c" can be seen to have considerable values when compared to the normal conditions and the two other phases [13].

Figure 4 depicts the maximum electromagnetic forces for the AC-supplied windings of the simulated FCL in a cycle after a fault with two types of winding arrangements. The average forces of the AC-supplied windings are calculated for the short-circuited phase "c", and when the forces of the two winding arrangements are compared, it can be derived that for short-circuited winding of distributed winding arrangement, the maximum radial and axial forces had about $24 \%$ and $70 \%$ decrease in magnitude, respectively, relative to those of a conventional winding arrangement.

It is important to note that all of the force magnitudes that are listed in the above tables are related to a scaleddown lab FCL with very smaller dimensions, power rating, and short-circuit current rating than industrial units in a real power network. At an industrial scale, the shortcircuit currents are hundreds or even thousands of times larger, and therefore, the forces are much greater than the values calculated in this paper. In all, the values in this paper are mentioned only to compare them to other winding arrangements, and these are not real values in a real power network.

\section{Conclusions}

This paper investigates distributed winding arrangements in terms of the axial and radial forces during the shortcircuit conditions of a three-phase FCL. The axial and radial forces of the FCL are calculated by using the finite element method to analyze a two-dimensional FCL model. A short-circuit current with a magnitude of $395 \mathrm{~A}$ is applied to the phase winding " $\mathrm{c}$ ", and the axial and radial forces of the AC-supplied windings are calculated in a transient state. For comparison, these forces are compared with those obtained from the results of a simulation of a conventional winding arrangement in which the phase windings are placed next to each other. The comparison showed that the distributed winding arrangement mitigated the average radial and axial forces by about $24 \%$ and $70 \%$, respectively, in the short-circuit condition. This indicates that the distributed winding arrangement is an appropriate choice for FCL devices and that this type of winding arrangement not only leads to a considerable decrease in the forces, but also can be realized with minimum production costs.

\section{References}

[1] Kalsi, Swarn S. and Alex Malozemoff, Power Engineering Society General Meeting 2, 1426 (2004).

[2] Y. Xin, W. Gong, X. Niu, Z. Cao, J. Zhang, B. Tian, H. Xi, Y. Wang, H. Hong, Y. Zhang, B. Hou, and X. Yang, IEEE Trans. Appl. Supercond. 17, 1760 (2007).

[3] Abdul Rahman, T. T. Lie, and K. Prasad, IEEE Trans. Appl. Supercond. 22, 5501211 (2012).

[4] Abdul Rahman, T. T. Lie, and K. Prasad, IEEE Trans. Appl. Supercond. 22, 5500108 (2012).

[5] Nadim Mahomed, Energize 12, 36 (2011).

[6] G. B. Kumbhar and S. V. Kulkarni, IEEE Trans. Power Delivery 22, 936 (2007).

[7] G. P. Peter, International Journal of Advancements in Technology 2, 142 (2011).

[8] Jawad Faiz, Bashir Mahdi Ebrahimi, and Tahere Noori, IEEE Trans. Magn. 44, 590 (2008).

[9] H. M. Ahn, J. Y. Lee, J. K. Kim, Y. H. Oh, S. Y. Jung, and S. C. Hahn, IEEE Trans. Ind. Appl. 47, 1267 (2011).

[10] Heydari, Hossein, and Faramarz Faghihi, IEEE Trans. Appl. Supercond. 20, 2276 (2010).

[11] S. L. Ho, Y. Li, Ho-ching Chris Wong, S. H. Wang, and R. Y. Tang, IEEE Trans. Magn. 40, 687 (2004).

[12] G. Bertagnolli, ABB Ltd. (2007).

[13] Hyun-Mo Ahn, Yeon-Ho Oh, Joong-Kyoung Kim, JaeSung Song, and Sung-Chin Hahn, IEEE Trans. Magn. 48, 819 (2012). 